\title{
Genetics of CFTR and male infertility
}

\author{
Jared M. Bieniek ${ }^{1}$, Craig D. Lapin ${ }^{2,3}$, Keith A. Jarvi ${ }^{4}$ \\ ${ }^{1}$ Tallwood Urology \& Kidney Institute, Hartford HealthCare, Hartford, CT, USA; ${ }^{2}$ Division of Pediatric Pulmonology, Connecticut Children's \\ Medical Center, Hartford, CT, USA; ${ }^{3}$ Department of Pediatrics, University of Connecticut, Farmington, CT, USA; ${ }^{4}$ Division of Urology, \\ Department of Surgery, Mount Sinai Hospital and Institute of Medical Sciences, University of Toronto, Toronto, ON, Canada \\ Contributions: (I) Conception and design: JM Bieniek, KA Jarvi; (II) Administrative support: None; (III) Provision of study materials or patients: \\ None; (IV) Collection and assembly of data: All authors; (V) Data analysis and interpretation: All authors; (VI) Manuscript writing: All authors; \\ (VII) Final approval of manuscript: All authors. \\ Correspondence to: Jared M. Bieniek, MD. Tallwood Urology \& Kidney Institute, 85 Seymour Street, \#416, Hartford, CT 06106, USA. \\ Email: Jared.bieniek@hhchealth.org.
}

\begin{abstract}
Cystic fibrosis (CF) is a rare autosomal-recessive disorder manifested as multisystem organ dysfunction. The cystic fibrosis transmembrane conductance regulator (CFTR) protein functions as an ion transporter on the epithelium of exocrine glands, regulating secretion viscosity. The CFTR gene, encoded on chromosome 7, is required for the production and trafficking of the intact and functional CFTR protein. Literally thousands of human CFTR allelic mutations have been identified, each with varying impact on protein quality and quantity. As a result, individuals harboring CFTR mutations present with a spectrum of symptoms ranging from CF to normal phenotypes. Those with loss of function but without full CF may present with CFTR-related disorders (CFTR-RDs) including male infertility, sinusitis, pancreatitis, atypical asthma and bronchitis. Studies have demonstrated associations between higher rates of CFTR mutations and oligospermia, epididymal obstruction, congenital bilateral absence of the vas deferens (CBAVD), and idiopathic ejaculatory duct obstruction (EDO). Genetic variants are detected in over three-quarters of men with CBAVD, the reproductive abnormality most classically associated with CFTR aberrations. Likewise, nearly all men with clinical CF will have CBAVD. Current guidelines from multiple groups recommend CFTR screening in all men with clinical CF or CBAVD though a consensus on the minimum number of variants for which to test is lacking. CFTR testing is not recommended as routine screening for men with other categories of infertility. While available CFTR panels include 30 to 96 of the most common variants, complete gene sequencing should be considered if there is a high index of suspicion in a high-risk couple (e.g., partner is CFTR mutation carrier). CF treatments to date have largely targeted end-organ complications. Novel CFTR-modulator treatments aim to directly target CFTR protein dysfunction, effectively circumventing downstream complications, and possibly preventing symptoms like vasal atresia at a young age. Future gene therapies may also hold promise in preventing or reversing genetic changes that lead to CF and CFTR-RD.
\end{abstract}

Keywords: Cystic fibrosis transmembrane conductance regulator protein (CFTR protein); congenital absence of vas deferens; cystic fibrosis (CF); CFTR

Submitted Dec 08, 2019. Accepted for publication Apr 01, 2020.

doi: $10.21037 /$ tau.2020.04.05

View this article at: http://dx.doi.org/10.21037/tau.2020.04.05

\section{Introduction}

The CFTR (cystic fibrosis transmembrane conductance regulator) gene, encoded on the long arm of chromosome 7 (7q31), consists of 27 coding exons spanning $230 \mathrm{~kb}(1)$.
The transcribed $6.5-\mathrm{kb}$ mRNA is translated into a 1,480 amino acid protein, the sequence of which directly affects protein folding and is crucial to proper functioning. Genetic alterations in CFTR can affect the final protein product in 
Table 1 CFTR mutation classes

\begin{tabular}{lllll}
\hline Class & CFTR defect & Worldwide frequency & Common mutations & Phenotype severity \\
\hline 1 & Protein synthesis & $10 \%$ (Ashkenazi 50\%) & G542X, W1282X & Severe \\
2 & Protein processing & $70 \%$ & F508del, N1303K & Severe \\
3 & Gating & $2-3 \%$ & G551D, R560T & Severe \\
4 & Conduction & Uncertain $(<2 \%)$ & R117H, R347P & Mild \\
5 & Reduced quantity & Uncertain $(<1 \%)$ & $3349+10 \mathrm{kbC}>$ G, IVS8-5T & Mild \\
6 & Reduced stability & Uncertain & Q1411X & Mild \\
\hline
\end{tabular}

a myriad of ways, from altered functionality to decreased production or combinations thereof (2).

Properly configured, CFTR acts as a cyclic AMP-activated ion channel, driving bicarbonate and chloride exchange and thus leading to water movement and decreased secretion viscosity in many organ systems $(2,3)$. Secretion viscosity is central to the physiologic roles of the lungs, exocrine pancreas, gastrointestinal system, and male reproductive tract. Significant biallelic CFTR abnormalities result in cystic fibrosis (CF), a severe disorder comprising symptoms in several or all of the organs noted above. Impaired chloride reabsorption in sweat glands also leads to the classic "salty sweat" found in patients with CF.

Only certain CFTR mutations manifest as CF. Less severe mutations or variants can present with limited signs or symptoms. Of particular interest to male infertility specialists, men with CF or CFTR-related disorders (CFTR-RDs) may manifest a spectrum of sperm obstructive processes, ranging from decreased to absent sperm counts. The most classically cited male infertility CFTR-RD is congenital bilateral absence of the vas deferens (CBAVD).

These phenotypes will obviously affect reproductive treatment options but should also spur genetic evaluation and counseling. With CFTR mutation carrier rate up to $4 \%$ in some American populations, the threshold for genetic testing should be low when evaluating men with any reproductive tract abnormalities (4). This review will serve to describe CFTR abnormalities, variable phenotypic presentations, genetic diagnostic methods, and treatment options with specific focus on relationships to male infertility. Sperm retrieval and utilization in men with obstructive azoospermia (OA) secondary to CFTR mutations are discussed in another article in this issue.

\section{Diseases associated with CFTR dysfunction}

\section{CFTR variants}

The Cystic Fibrosis Mutation Database, maintained by The Hospital for Sick Children in Toronto, Canada, is charged with keeping an up-to-date listing of identified CFTR mutations (5). As of the writing of this manuscript, 2,074 mutations are currently detailed, each of varying prevalence. The nomenclature of each variant was updated in 2010 to the standards recommended by the Human Genome Variation Society. In order to retain relevance to the body of literature on the topic, this article will utilize conventional CFTR mutation designations.

Researchers have classified CFTR mutations into six different classes: protein synthesis (Class 1), protein processing (Class 2), gating (Class 3), conduction (Class 4), reduced quantity (Class 5), and reduced stability (Class 6) defects as detailed in Table $1(2,6,7)$. Each functional class may be caused by various genetic aberrations including base deletion, substitution, insertion, or complete gene duplication or deletion. The F508del mutation, for example, the most common CF abnormality, represents a protein processing (Class 2) mutation due to a three-nucleotide deletion, removing a crucial phenylalanine amino acid (6). Without it, CFTR protein cannot fold properly resulting in rapid degradation. While classically considered a Class 2 (protein processing) mutation, F508del also results in gating defects (Class 3) and reduced protein stability (Class 6) (7). CFTR mutations may more appropriately be considered part of a complex Venn diagram as discussed in more recent reviews.

Another common variant site is the polythymidine tract at the terminal end of intron 8 . Repeat thymidine bases influence the splicing of exon 9 with inversely proportionate 
splicing to the number of bases. Thus, $7 \mathrm{~T}$ or $9 \mathrm{~T}$ sequences generally result in normal exon 9 splicing while the shorter 5T variant (also known as IVS8-5T, a Class V mutation) results in frequent exon 9 skipping, leading to a reduced quantity of functional CFTR protein (8). This variant does not cause classic CF, but may increase the severity of CF among those with two CFTR gene mutations or reduce functional CFTR protein leading to CFTR-RDs. With an allele frequency of $5 \%$, the carrier frequency of $5 \mathrm{~T}$ variants is approximately $10 \%$ in the general population $(9,10)$.

The Clinical and Functional TRanslation of CFTR project (CFTR2), supported by the US Cystic Fibrosis Foundation, details unique CFTR variants and the propensity of each for functional impairment (11). Variants are characterized as CF-causing, variants of varying clinical consequence, non-CF, and those of unknown significance. Homozygous expression of F508del is classically associated with a severe CF phenotype. Varying genotypic combinations of the variant categories noted above however lead to a greater spectrum of CF and CFTR-RD phenotype severity.

\section{Phenotypes of CFTR dysfunction}

Contrary to many genetic disorders, CFTR mutations are not reflected by an "all or none" disease state. The phenotypes of CF and CFTR-RDs, rather, are similar, but vary based on the number and severity of organ systems involved. Symptoms can range from multisystem organ failure with CF, through more mild CFTR-RDs, to no symptoms whatsoever due to differential tissue CFTR expressivity and sensitivity. Most CBAVD patients, even those with two identified CFTR variants, have no lung disease, indicating the relative sensitivity of the vasa to CFTR dysfunction. This variability is believed to be secondary to tissue-specific alternative splicing which occurs more in the vasa compared to other tissues (12). Likewise complicating the picture, CFTR variants have heterogeneous effects on protein levels and functionality. Phenotypic symptom severity can even vary among individuals with identical CFTR genotypes secondary to other gene modifiers (1).

The impairment in CFTR protein quantity or quality varies widely based on genetic mutation class and type. The combination of two CFTR alleles ultimately determines the presence or absence of symptoms and disease severity. Classically two pathogenic CF-causing mutations, such as F508del, lead to CF. The combination of one CF-causing and one non-CF-causing mutation, a compound heterozygote, often allows the CFTR gene to retain some level of functionality. Individuals with these permutations can overlap with the CF phenotype, leading to possible male infertility with a small percentage reporting mild CF symptoms and bringing into question possible subclinical effects (13). Pulmonary evaluation of eight men with isolated CBAVD and CFTR mutations by Gilljam et al. revealed higher rates of inflammatory markers and opportunistic bacteria compared to controls (14). Appropriate referrals should be made for men presenting with CFTR-related infertility and any abnormal pulmonary signs or symptoms.

\section{Cystic fibrosis}

$\mathrm{CF}$, as noted, is caused by significant aberrations in CFTR protein function. As a result, epithelial secretions are pathologically viscous, resulting in multisystem disease affecting the respiratory tract, exocrine pancreas, gastrointestinal system, and male reproductive tract. Clinical symptoms include chronic cough, recurrent sinusitis, obstructive lung disease, recurrent pulmonary infections, and male infertility (1). Pulmonary disease remains the major cause of CF morbidity and mortality (15). With advanced care however, life expectancy has increased significantly. According to the 2017 CF Patient Registry Annual Data Report, the current median survival is 43.6 years compared to 32.7 years as recently as 2002 (16). Now that men with CF are living well into and beyond their reproductive years, infertility care is coming to the forefront but the challenge remains that essentially all have bilateral vas deferens absence.

\section{CFTR-RDs}

A subset of individuals may present with genetically related, or CFTR-RDs, without meeting full criteria for CF (17). The spectrum of phenotypic presentations of these individuals with varying CFTR mutations (often heterozygous) is reflective of the complex landscape of genotypic subtypes. Relevant to this review, men with CFTR abnormalities can have isolated infertility with a range of sperm transport obstructive processes as described in Table 2. The central theme is a higher relative frequency of severe compared to mild CFTR mutations in men with CF trending progressively downwards in men with CBAVD, congenital unilateral absence of the vas deferens (CUAVD), epididymal obstruction, and oligospermia.

The most classic reproductive tract abnormality 
Table 2 Potential reproductive tract abnormalities in men with CFTR abnormalities

\begin{tabular}{lll}
\hline Reproductive tract abnormality & CFTR abnormality prevalence* & $\begin{array}{l}\text { Severity of CFTR abnormality } \\
\text { (allele 1; allele 2) }\end{array}$ \\
\hline Oligospermia & $17.5 \%(18)$ & Mild; wild \\
Epididymal obstruction & $34-47 \%(9,19)$ & Severe/mild; wild \\
Ejaculatory duct obstruction and seminal vesicle abnormalities ${ }^{\dagger}$ & $86 \%(20)$ & Severe/mild; mild/wild \\
CUAVD & $46 \%(21)$ & Severe/mild; mild/wild \\
CBAVD & $78 \%(22)$ & Severe/mild; mild/wild \\
CF with CBAVD & $95 \%(23)$ & Severe; severe \\
\hline
\end{tabular}

${ }^{*}$, one or more CFTR mutations; ${ }^{\dagger}$, EDO of idiopathic etiology; Wild denotes wild-type allele or no detectable mutation. CUAVD, congenital unilateral absence of the vas deferens; CBAVD, congenital bilateral absence of the vas deferens; EDO, ejaculatory duct obstruction.

associated with CFTR mutations, isolated CBAVD, is often initially detected at the time of urology evaluation for male infertility. Clinical exam typically reveals complete absence of bilateral vas deferens and distal epididymis with seminal vesicle hypoplasia or absence (24). CBAVD represents the only male infertility-causing genetic mutation that can be detected on physical examination. Laboratory testing will demonstrate OA with normal gonadotropins and reproductive hormones. Comprehensive semen analysis will typically reveal no sperm with low ejaculate volume $(<1 \mathrm{~mL})$, low $\mathrm{pH}(<7)$, and reduced fructose level (25).

Bilateral vasal absence is highly suggestive of CFTR allelic abnormalities. In a 2012 meta-analysis by Yu et al., $78 \%$ of men with CBAVD were noted to have at least one identified CFTR mutation (22). Delving deeper, $46 \%$ of men in the included studies had biallelic mutations and $28 \%$ had a single mutation. A criticism of the review is that many of the individual studies were conducted in earlier years when fewer CFTR mutations were known and detectable (26). Among those men with heterozygous mutations, the most common pairing was F508del/IVS8-5T, comprising 17\% of CBAVD cases. All other combinations were found in less than $3 \%$ of cases. While conventional thinking suggests that CBAVD is caused by biallelic variants, the data above which includes studies with exhaustive CFTR screening methods (that theoretically would capture any $C F T R$ variant) clearly demonstrates a subset of CBAVD men with a wild type allele, obligatorily trans to a CFTR variant (9). In these men, there are clearly other genetic regions or modifiers reducing CFTR protein quantity or quality and resulting in vasal atresia (27).

As if not heterogeneous enough, some men with congenital absence of the vas deferens have completely normal CFTR genotypes (two wild-type alleles). In these cases, vasal absence is thought to be secondary to alternative genetic or toxic insults affecting Wolffian duct development. To review, the rudimentary embryologic Wolffian duct divides into two limbs near seven weeks of development. One limb forms the basis for ureteral and renal development while the other is responsible for much of the sperm transport system, namely the distal two-thirds of the epididymis, vas deferens, and seminal vesicle (28). Any insult to the Wolffian duct prior to seven weeks then results in abnormalities to the male reproductive structures in addition to the ipsilateral kidney (29). In contrast, renal development is typically normal in cases of CBAVD, suggesting that vasal atresia in men often harboring CFTR mutations occurs after the critical seven-week time point. A single center review of over a decade of men with CBAVD revealed only 17 of $168(10.1 \%)$ men with unilateral renal agenesis (URA) (29). Schlegel et al. reported a similar $11 \%$ rate of URA in 84 men presenting with CBAVD (30). While these rates are low, the incidence of URA in CBAVD is certainly not negligible.

If a unilateral Wolffian duct is affected early during embryogenesis, specifically before seven weeks, vasal and renal development may be jointly affected leading to unilateral agenesis (29). In a meta-analysis reported in early 2019 by Cai et al., URA was detected in $22 \%$ of 141 men with CUAVD (21). Contrast this to the 10\% rate of URA in men with CBAVD noted above. Among men with CUAVD, $46 \%$ had one or more CFTR mutations (one detected in $27 \%$ and two in $5 \%)$. The most frequently abnormal alleles were IVS8-5T (9\%) and F508del (4\%). Interestingly, the aggregate frequency of F508del/IVS8-5T, the most common heterozygous genotype in CBAVD, was $0 \%$ in 
men with CUAVD. It remains unknown why some $C F T R$ mutations cause CUAVD while others lead to CBAVD. Future research may also help elucidate alternative genetic etiologies of CUAVD.

Given the association between vasal and renal development and maldevelopment, the current American Urological Association (AUA) Best Practice Statement on evaluation of azoospermic men recommends offering screening renal ultrasound to men with CUAVD or men with CBAVD and no identified CFTR abnormalities (31). The apparent subpopulation excluded from this recommendation is those with CBAVD and an identified CFTR variant. McCallum et al. reported a single center series including 17 men with URA and CBAVD, only three of which had a CFTR mutation (29). Cases of URA should be advised of solitary kidney precautions and annual follow up recommended for blood pressure, serum creatinine, and urinalysis monitoring.

Sperm obstructive processes at various levels and severity other than congenital vasal absence have additionally been linked to CFTR abnormalities. In 62 azoospermic men, Jarvi et al. detected CFTR variants in 20/25 (80\%) men with CBAVD, 8/17 (47\%) with OA, and only 2/18 (11\%) with nonobstructive azoospermia (19). All men with OA were noted to have epididymal obstruction at the time of surgical sperm retrieval with palpable vasa. EDO, typically a result of prostatic surgery, infection, or cysts, has also been associated with CFTR mutations. In a small series of men with idiopathic bilateral EDO with abnormal seminal vesicles, 6/7 (86\%) were found to have CFTR variants (20). There did not appear to be a connection between genetic variants and isolated seminal vesicle abnormalities in the same series.

Despite studies suggesting direct CFTR involvement in spermatogenesis, the prevalence of $C F T R$ mutations among men with nonobstructive azoospermia ( $\mathrm{n}=5 / 45$, one F508del and four IVS8-5T) was not significantly different than that observed in the general population (32). Oligospermia, or reduced sperm number, on the other hand was noted in $14 / 80(17.5 \%)$ men, a higher than expected frequency compared to the local population (18). While some have used this data to support the hypothesis that CFTR impacts spermatogenesis, it may more accurately reflect partial obstruction, another aspect of the CFTR phenotypic spectrum. The data supporting broad associations between CFTR mutations and various male reproductive tract abnormalities would suggest a low threshold for genetic testing.

\section{Prevalence of CFTR variants and associated diseases}

$\mathrm{CF}$ is an autosomal recessive disorder requiring maternal and paternal transmission of pathogenic CFTR mutations. Just as CFTR gene mutations vary by ethnic and racial background, so does CF prevalence. Incidence of CF is as high as 1 in 1,600-2,000 individuals of northern European descent $(24,33)$. As such, CF represents the most common Caucasian life-limiting genetic defect (1). The reported rate of CF in other groups is much lower, affecting 1:15,000 African Americans and 1:31,000 Asian Americans (34). Overall, it is estimated that 30,000 individuals are currently living with CF in the United States.

The F508del mutation is the most common pathogenic allele identified in CF patients worldwide, detected in $66 \%$ of cases (35). Similar to the variability in CF disease, CFTR genotypic variants differ widely based on ethnicity and geographic location. Among individuals with CF, F508del is detected in $100 \%$ of Faroe Islanders, $85 \%$ of North Americans, 70-80\% of northern Europeans, 50\% of southern Europeans, 48\% of African Americans, 46\% of US Hispanics, $30 \%$ of Ashkenazi Jews, and rarely in American Indians $(16,36,37)$. An alternative mutation, W1282X, surpasses F508del as the most common mutation in Ashkenazi Jews with CF.

As the frequency of clinical CF is relatively high so far as genetic disorders go, the carrier frequency is expectedly high, as high as 1 in 28 Americans of Northern European descent (4). Carrier frequency varies again by ethnic and racial background, being detected in 1 in 29 Ashkenazi Jews, 1 in 61 African Americans, and 1 in 118 Asian Americans $(4,38,39)$. As such, the ethnicity of partners of men with CFTR-related infertility must be considered when predicting risk of mutations in future offspring.

CFTR variants have been clearly linked to obstructive male infertility processes as noted above $(9,18-22)$. CBAVD is present in $95-98 \%$ of men with CF (40). Various reports cite the incidence of CBAVD as high as $1-2 \%$ of infertile men $(1,41)$. CUAVD, which is less frequently associated with CFTR abnormalities, is estimated to occur in less than $1 \%$ of the population (42). A disconnect appears when compiling $C F T R$ variant and congenital absence of the vas deferens data. Despite a CFTR carrier frequency among North American Caucasians of 4-5\%, CBAVD is diagnosed in only $1-2 \%$ of infertile men and certainly less than $1 \%$ of the general male population $(1,41,43)$. More simply put, most men with CFTR mutations have normal bilateral vasa. 
Fitting these facts together suggests a genetic "dosing level" required for normal Wolffian duct development, below which absence of the vas deferens occurs.

\section{Diagnostic approach}

Initial evaluation of any infertile male begins with a history and physical examination followed by laboratory testing including comprehensive semen analysis and serum hormones (44). Often a semen analysis will be done before men are referred to a male infertility specialist. When low volume azoospermia is found, careful attention must be paid to the presence or absence of bilateral vasa and epididymides on exam. Even if CBAVD is detected, it is important not to ignore the other components of male testing, namely serum hormones, as up to $12 \%$ of men with CBAVD have been shown to have impaired spermatogenesis in addition to their obstructive process (45).

Genetic and functional testing form the gold standard for diagnosing CF and CFTR-RDs. CF is defined by one or more characteristic clinical features and evidence of abnormality in CFTR function such as biallelic pathogenic CFTR mutations, two abnormal quantitative sweat chloride tests, or transepithelial nasal potential difference characteristic of CF (1). Diagnosis can be confirmed in infants with elevated trypsinogen on newborn screening and findings of biallelic CFTR mutations or elevated sweat chloride test. Individuals presenting with findings or symptoms suggestive of a CFTR-RD should also undergo CFTR testing.

Multiple society guidelines, including those from the World Health Organization, American Society of Reproductive Medicine, and AUA, recommend CFTR mutation analysis in all men with CF or $\operatorname{CBAVD}(31,33,46)$. When reviewed, the literature contains a high strength of evidence to support these statements (33). Despite the higher prevalence of CFTR mutations and variants in men with epididymal obstruction and oligospermia, routine mutation analysis has not been recommended for these categories of infertility to date. The AUA Best Practice Statement "The Evaluation of the Azoospermic Male", last reviewed in 2011, recommends "testing for cystic fibrosis transmembrane conductance regulator abnormalities [that] should include at minimum a panel of common point mutations and the $5 T$ allele" (31). A consensus on the minimum number of variants for which to test is lacking. In cases of high risk to offspring, namely in men with CBAVD with negative CFTR panel testing and a CFTR carrier partner, complete gene sequencing should be considered.

Current CFTR mutation testing is typically done by searching for panels of predetermined high-risk or highfrequency mutations. Most CFTR panels will include 30 to 96 of the most common mutations. Contrast this to the over 2,000 mutations identified in the CF Mutation Database (5). Our own institution's CFTR test offerings include a CF screen (32 mutations), CF expanded screen (161 mutations), $\mathrm{CF}$ intron 8 poly- $\mathrm{T}$ analysis, and CF complete rare mutation analysis or entire gene sequence. The common variants included in the 32-mutation screening panel including their relative frequency in those with CF are depicted in Table 3 (35). Testing is performed via a multiplex polymerase chain reaction using specific primers for each genetic variant. Multigene panels at other laboratories may employ alternative methods such as multiplex ligation-dependent probe amplification or gene-targeted microarray (1).

Failure to identify a variant on CFTR mutation panel testing in a man with CBAVD should not be misinterpreted as ruling out a mutation. In a series of 64 men with CBAVD, $46 \%$ had detectable mutations missed by a 31-mutation panel which was decreased to $17 \%$ missed when including IVS8-5T analysis (9). The same study reported undetected mutations in up to $58 \%$ of men with idiopathic EDO using the panel and IVS8-5T testing. For purposes of reproductive planning, it should be assumed that all men with CBAVD harbor a CFTR abnormality, therefore negative testing only suggests the absence of the most common mutations. Expanded panels, entire gene sequencing, and thorough partner testing should be considered if the index of suspicion is high.

Diagnosis of CFTR mutations offers several potential benefits. Referral to a center specializing in CF care should be considered for a full assessment of CFTR dysfunction phenotypes. In patients with mild CF symptoms such as sinusitis not previously recognized to be CFTR-related, alternative therapeutic management options may be offered. Patient testing also provides important genetic information for siblings. Siblings of a man with CBAVD with biallelic CFTR mutations have a $75 \%$ chance of harboring at least one mutation inherited from their parents.

Partner testing also deserves special attention. When planning assisted reproductive techniques for men with known or suspected CFTR abnormalities, female partner screening should be offered $(31,46)$. If the female partner is found to be a carrier, preimplantation genetic diagnosis is useful to determine risk of CF and CBAVD to future progeny. Genetic counselling should be recommended as 
Table 3 Frequency of CFTR mutations in 32-mutation CF screening panel (note intron 8 poly- $T$ analysis not included)

\begin{tabular}{|c|c|c|}
\hline Mutation & $\begin{array}{l}\text { Relative } \\
\text { frequency (\%) }\end{array}$ & $\begin{array}{l}\text { Population with highest } \\
\text { prevalence }\end{array}$ \\
\hline F508del & 66.0 & Northern European \\
\hline G542X & 2.4 & Ashkenazi Jewish, Spanish \\
\hline G551D & 1.6 & English \\
\hline N1303K & 1.3 & Italian \\
\hline W1282X & 1.2 & Ashkenazi Jewish \\
\hline R553X & 0.7 & German \\
\hline $621+1 \mathrm{G}>\mathrm{T}$ & 0.7 & French-Canadian \\
\hline $1717-1 \mathrm{G}>\mathrm{A}$ & 0.6 & Italian \\
\hline $\mathrm{R} 117 \mathrm{H}$ & 0.3 & - \\
\hline R1162X & 0.3 & Italian \\
\hline R347P & 0.2 & - \\
\hline $3849+10 \mathrm{kbC}>\mathrm{T}$ & 0.2 & - \\
\hline$\Delta \mathrm{I} 507$ & 0.2 & - \\
\hline 394delTT & 0.2 & Nordic, Finnish \\
\hline G85E & 0.2 & - \\
\hline R560T & 0.2 & - \\
\hline A455E & 0.1 & - \\
\hline 1078delT & 0.1 & - \\
\hline $2789+5 G>A$ & 0.1 & Spanish \\
\hline 3659delC & 0.1 & - \\
\hline R334W & 0.1 & - \\
\hline $1898+1 G>A$ & 0.1 & - \\
\hline $711+1 \mathrm{G}>\mathrm{T}$ & 0.1 & French-Canadian \\
\hline $2183 A A>G$ & 0.1 & Italian \\
\hline 3905insT & 0.1 & Amish, Acadian, Swiss \\
\hline S549N & 0.1 & - \\
\hline 2184delA & 0.1 & - \\
\hline $3120+1 G>A$ & - & - \\
\hline V520F & - & - \\
\hline 3876delA & - & - \\
\hline $\mathrm{R} 347 \mathrm{H}$ & - & - \\
\hline S549R & - & - \\
\hline
\end{tabular}

CF, cystic fibrosis. early as possible in these cases, both for male and female partners.

\section{Treatments and impact on male infertility}

Following CF diagnosis, treatment is guided by efficacyproven preventative and therapeutic algorithms. These treatments to date have largely targeted end-organ complications. Novel CFTR-modulator treatments aim to directly target CFTR protein dysfunction, effectively preventing downstream complications. Advancements in $\mathrm{CF}$ care have resulted in drastic improvements in patient survival and increase interest in assisted reproductive options (16).

Respiratory management is central to each CF patient's treatment plan. Individuals with CF may expect a sequence of inhaled medications including bronchodilators, hypertonic saline, dornase alfa, aerosolized antibiotics, and airway clearance techniques (1). Unfortunately, no current treatments exist to reverse the vasal atresia associated with CBAVD. Options for retrieval and utilization of sperm from men with CFTR mutations are discussed in a later article in this issue.

CFTR-modulator therapies, approved in 2012, are the first to directly target CFTR protein dysfunction rather than downstream organ involvement. These therapies currently fall into two approved categories (potentiators and correctors) with a third (amplifiers) being actively investigated. The theory behind potentiators and correctors is that altering the defects in the CFTR channel will allow some level of functional normalization leading to clinical benefit (47). Potentiators hold the CFTR chloride channel in an open position, thus any CFTR mutation that allows protein to reach the cell surface (Class 3 and some Class 4) will have improved ion conductance. Correctors have been designed to address F508del mutations specifically by binding to misshapen CFTR proteins, improving the three-dimensional structure and acting as chaperones to avoid elimination by the endoplasmic reticulum. Combining potentiator and single corrector therapies has shown significant but clinically limited improvement in patients with F508del mutations. Recent studies show that a combination of two correctors plus a potentiator, or triple therapy, provides a more significant clinical response (48). An additional class of modulator therapy, known as amplifiers, is now undergoing phase 3 studies. It is proposed that 
amplifiers selectively increase the amount of abnormal CFTR protein thus providing additional substrate upon which correctors and potentiators can act (49).

It is interesting to speculate how CFTR-modulator therapy may affect male infertility in CF and CBAVD. When starting potentiators early (6-12 months of age), results suggest improved pancreatic function that was previously felt to be irreversible (50). Pancreatic insufficiency is hypothesized to occur in utero due to plugging of pancreatic exocrine ducts and autolysis. Although CBAVD is found in nearly all adult males with CF, it is less complete in children, raising the question whether infertility may lessen as modulator therapies become more pervasive and are initiated at earlier and earlier ages (40).

With FDA approval of the new gene therapy Zolgensma (onasemnogene abeparvovec-xioi) in May 2019 for infants with spinal muscular atrophy, we enter an exciting time in the treatment of genetic disorders altogether (51). Rather than responding to disease complications, future gene therapies carry the potential to prevent or reverse genetic changes leading to CF and CFTR-RD. With groundbreaking treatments, though, comes mind-boggling price tags. In the case of Zolgensma, the one-time treatment will cost over two million USD. The pathway to effective gene therapy for other diseases other than spinal muscular atrophy may not be smooth, though. CF and CBAVD represent phenotypic expressions of literally thousands of different CFTR mutations. Gene therapies for CF are in early clinical trials but have yet to show clinical effectiveness $(1,52)$. It will be interesting to see how we manage the intersection of amazing technology, sky-rocketing costs, and swelling healthcare costs as future gene therapies and other CFTR-related treatments are developed.

\section{Conclusions}

CFTR abnormalities comprise a heterogeneous group of genetic mutations that have variable effects on protein quantity and functionality, manifested by inconsistent disease penetrance. Involved in the regulation of secretion viscosity, CFTR mutations can lead to CF and CFTR$\mathrm{RDs}$ including male reproductive tract abnormalities and infertility. The severity of reproductive tract changes varies with both the genotype and the frequency of CFTR mutations. Studies have demonstrated gene abnormalities in almost all CF patients, approximately $80 \%$ of men with CBAVD, $46 \%$ of CUAVD patients, $33-47 \%$ of men with epididymal obstruction, and $17.5 \%$ of men with oligospermia. A higher relative frequency of severe compared to mild CFTR mutations exists in men with CF trending progressively downwards in men with CBAVD, CUAVD, epididymal obstruction, and oligospermia. Men with CF and CBAVD have significantly higher chances of having children with classic $\mathrm{CF}$, thus these men and their partners should be screened for CFTR mutations.

\section{Acknowledgments}

Funding: None.

\section{Footnote}

Provenance and Peer Review: This article was commissioned by the Guest Editors (Keith Jarvi and Jared Bieniek) for the series "Genetic Causes and Management of Male Infertility" published in Translational Andrology and Urology. The article was sent for external peer review organized by the Guest Editors and the editorial office.

Conflicts of Interest: All authors have completed the ICMJE uniform disclosure form (available at http://dx.doi. org/10.21037/tau.2020.04.05). The authors have no conflicts of interest to declare. The series "Genetic Causes and Management of Male Infertility" was commissioned by the editorial office without any funding or sponsorship.

Ethical Statement: The authors are accountable for all aspects of the work in ensuring that questions related to the accuracy or integrity of any part of the work are appropriately investigated and resolved.

Open Access Statement: This is an Open Access article distributed in accordance with the Creative Commons Attribution-NonCommercial-NoDerivs 4.0 International License (CC BY-NC-ND 4.0), which permits the noncommercial replication and distribution of the article with the strict proviso that no changes or edits are made and the original work is properly cited (including links to both the formal publication through the relevant DOI and the license). See: https://creativecommons.org/licenses/by-nc-nd/4.0/.

\section{References}

1. Ong T, Marshall SG, Karczeski BA, et al. Cystic Fibrosis and Congenital Absence of the Vas Deferens. In: Adam MP, Ardinger HH, Pagon RA et al. editors. 
GeneReviews® [Internet]. Seattle (WA): University of Washington, Seattle, 1993-2020.

2. Welsh MJ, Smith AE. Molecular mechanisms of CFTR chloride channel dysfunction in cystic fibrosis. Cell 1993;73:1251-4.

3. Borowitz D. CFTR, bicarbonate, and the pathophysiology of cystic fibrosis. Pediatr Pulmonol 2015;50 Suppl 40:S24-30.

4. Hamosh A, FitzSimmons SC, Macek M, Jr., et al. Comparison of the clinical manifestations of cystic fibrosis in black and white patients. J Pediatr 1998;132:255-9.

5. Cystic Fibrosis Centre at the Hospital for Sick Children. Cystic Fibrosis Mutation Database: CFMDB Statistics. Available online: http://genet.sickkids.on.ca/StatisticsPage. html. Accessed October 20, 2019.

6. Rogan MP, Stoltz DA, Hornick DB. Cystic fibrosis transmembrane conductance regulator intracellular processing, trafficking, and opportunities for mutationspecific treatment. Chest 2011;139:1480-90.

7. Veit G, Avramescu RG, Chiang AN, et al. From CFTR biology toward combinatorial pharmacotherapy: expanded classification of cystic fibrosis mutations. Mol Biol Cell 2016;27:424-33.

8. Viel M, Leroy C, Des Georges M, et al. Novel length variant of the polypyrimidine tract within the splice acceptor site in intron 8 of the CFTR gene: consequences for genetic testing using standard assays. Eur J Hum Genet 2005;13:136-8.

9. Mak V, Zielenski J, Tsui LC, et al. Proportion of cystic fibrosis gene mutations not detected by routine testing in men with obstructive azoospermia. JAMA 1999;281:2217-24.

10. Groman JD, Hefferon TW, Casals T, et al. Variation in a repeat sequence determines whether a common variant of the cystic fibrosis transmembrane conductance regulator gene is pathogenic or benign. Am J Hum Genet 2004;74:176-9.

11. US Cystic Fibrosis Foundation. In: Clinical and Functional Translation of CFTR. Available online: https://www.cftr2. org/. Accessed October 12, 2019.

12. Cuppens H, Cassiman JJ. CFTR mutations and polymorphisms in male infertility. Int J Androl 2004;27:251-6.

13. Dörk T, Dworniczak B, Aulehla-Scholz C, et al. Distinct spectrum of CFTR gene mutations in congenital absence of vas deferens. Hum Genet 1997;100:365-77.

14. Gilljam M, Moltyaner Y, Downey GP, et al. Airway inflammation and infection in congenital bilateral absence of the vas deferens. Am J Respir Crit Care Med 2004;169:174-9.

15. Wilschanski M, Dupuis A, Ellis L, et al. Mutations in the cystic fibrosis transmembrane regulator gene and in vivo transepithelial potentials. Am J Respir Crit Care Med 2006;174:787-94.

16. Cystic Fibrosis Foundation. In: 2017 Patient Registry Annual Data Report. Available online: https://www.cff.org/ Research/Researcher-Resources/Patient-Registry/2017Patient-Registry-Annual-Data-Report.pdf. Accessed October 12, 2019.

17. Bombieri C, Claustres M, De Boeck K, et al. Recommendations for the classification of diseases as CFTR-related disorders. J Cyst Fibros 2011;10 Suppl 2:S86-102.

18. van der Ven K, Messer L, van der Ven H, et al. Cystic fibrosis mutation screening in healthy men with reduced sperm quality. Hum Reprod 1996;11:513-7.

19. Jarvi K, Zielenski J, Wilschanski M, et al. Cystic fibrosis transmembrane conductance regulator and obstructive azoospermia. Lancet 1995;345:1578.

20. Meschede D, Dworniczak B, Behre HM, et al. CFTR gene mutations in men with bilateral ejaculatory-duct obstruction and anomalies of the seminal vesicles. Am J Hum Genet 1997;61:1200-2.

21. Cai H, Qing X, Niringiyumukiza JD, et al. CFTR variants and renal abnormalities in males with congenital unilateral absence of the vas deferens (CUAVD): a systematic review and meta-analysis of observational studies. Genet Med 2019;21:826-36.

22. Yu J, Chen Z, Ni Y, et al. CFTR mutations in men with congenital bilateral absence of the vas deferens (CBAVD): a systemic review and meta-analysis. Hum Reprod 2012;27:25-35.

23. Chillón M, Casals T, Mercier B, et al. Mutations in the cystic fibrosis gene in patients with congenital absence of the vas deferens. N Engl J Med 1995;332:1475-80.

24. Hotaling J, Carrell DT. Clinical genetic testing for male factor infertility: current applications and future directions. Andrology 2014;2:339-50.

25. Attardo T, Vicari E, Mollica F, et al. Genetic, andrological and clinical characteristics of patients with congenital bilateral absence of the vas deferens. Int J Androl 2001;24:73-9.

26. Anguiano A, Oates RD, Amos JA, et al. Congenital bilateral absence of the vas deferens. A primarily genital form of cystic fibrosis. JAMA 1992;267:1794-7.

27. Havasi V, Rowe SM, Kolettis PN, et al. Association of 
cystic fibrosis genetic modifiers with congenital bilateral absence of the vas deferens. Fertil Steril 2010;94:2122-7.

28. Gibbons MD, Cromie WJ, Duckett JW, Jr. Ectopic vas deferens. J Urol 1978;120:597-604.

29. McCallum T, Milunsky J, Munarriz R, et al. Unilateral renal agenesis associated with congenital bilateral absence of the vas deferens: phenotypic findings and genetic considerations. Hum Reprod 2001;16:282-8.

30. Schlegel PN, Shin D, Goldstein M. Urogenital anomalies in men with congenital absence of the vas deferens. J Urol 1996;155:1644-8.

31. Jarow J, Sigman M, Kolettis PN, et al. The Evaluation of the Azoospermic Male. AUA Best Pratice Statement, 2011.

32. Mak V, Zielenski J, Tsui LC, et al. Cystic fibrosis gene mutations and infertile men with primary testicular failure. Hum Reprod 2000;15:436-9.

33. Barratt CLR, Bjorndahl L, De Jonge CJ, et al. The diagnosis of male infertility: an analysis of the evidence to support the development of global WHO guidancechallenges and future research opportunities. Hum Reprod Update 2017;23:660-80.

34. Rosenstein BJ, Cutting GR. The diagnosis of cystic fibrosis: a consensus statement. Cystic Fibrosis Foundation Consensus Panel. J Pediatr 1998;132:589-95.

35. Cystic Fibrosis Centre at the Hospital for Sick Children. Cystic Fibrosis Mutation Database: Most common CFTR mtuations in the world. Available online: http://www. genet.sickkids.on.ca/cftr/resource/Table1.html. Accessed Oct 20, 2019.

36. Lommatzsch ST, Aris R. Genetics of cystic fibrosis. Semin Respir Crit Care Med 2009;30:531-8.

37. Bobadilla JL, Macek M Jr, Fine JP, et al. Cystic fibrosis: a worldwide analysis of CFTR mutations--correlation with incidence data and application to screening. Hum Mutat 2002;19:575-606.

38. Kerem B, Chiba-Falek O, Kerem E. Cystic fibrosis in Jews: frequency and mutation distribution. Genet Test 1997;1:35-9.

39. Rohlfs EM, Zhou Z, Heim RA, et al. Cystic fibrosis carrier testing in an ethnically diverse US population. Clin Chem 2011;57:841-8.

40. Blau H, Freud E, Mussaffi H, et al. Urogenital abnormalities in male children with cystic fibrosis. Arch Dis Child 2002;87:135-8.

41. Jequier AM, Ansell ID, Bullimore NJ. Congenital absence of the vasa deferentia presenting with infertility. J Androl
1985;6:15-9.

42. Klapproth HJ, Young IS. Vasectomy, vas ligation and vas occlusion. Urology 1973;1:292-300.

43. Johnson JP, Louie E, Lewiston NJ, et al. beta-adrenergic sweat responses in cystic fibrosis heterozygotes with and without the delta F508 allele. Pediatr Res 1991;29:525-8.

44. Jarow J, Sigman M, Kolettis PN, et al. The Optimal Evaluation of the Infertile Male. AUA Best Practice Statement. 2011.

45. Meng MV, Black LD, Cha I, et al. Impaired spermatogenesis in men with congenital absence of the vas deferens. Hum Reprod 2001;16:529-33.

46. Practice Committee of the American Society for Reproductive Medicine in collaboration with the Society for Male R, Urology. Evaluation of the azoospermic male: a committee opinion. Fertil Steril 2018;109:777-82.

47. Grasemann H. CFTR Modulator Therapy for Cystic Fibrosis. N Engl J Med 2017;377:2085-8.

48. Keating D, Marigowda G, Burr L, et al. VX-445Tezacaftor-Ivacaftor in Patients with Cystic Fibrosis and One or Two Phe508del Alleles. N Engl J Med 2018;379:1612-20.

49. Downey D, Flume P, Jain M, et al. WS06-1 Initial results evaluating combinations of the novel CFTR corrector PTI-801, potentiator PTI-808, and amplifier PTI-428 in cystic fibrosis subjects. J Cyst Fibros 2019;18:S10.

50. Davies J, Wang LT, Panorchan P, et al. WS06-4 Ivacaftor (IVA) treatment in patients 6 to \&lt; 12 months old with cystic fibrosis with a CFTR gating mutation: results of a 2-part, single-arm, phase 3 study. J Cyst Fibros 2019;18:S11.

51. Food and Drug Administration. In: FDA approves innovative gene therapy to treat pediatric patients with spinal muscular atrophy, a rare disease and leading genetic cause of infant mortality. 2019. Available online: https://www.fda.gov/news-events/press-announcements/ fda-approves-innovative-gene-therapy-treat-pediatricpatients-spinal-muscular-atrophy-rare-disease. Accessed October 122019.

52. Prickett M, Jain M. Gene therapy in cystic fibrosis. Transl Res 2013;161:255-64.

Cite this article as: Bieniek JM, Lapin CD, Jarvi KA. Genetics of CFTR and male infertility. Transl Androl Urol 2021;10(3):13911400. doi: $10.21037 /$ tau.2020.04.05 